\title{
Traffic Density Analysis based on Image Segmentation with Adaptive Threshold
}

\author{
Luong Anh Tuan Nguyen \\ Ho Chi Minh City University of Transport \\ No. 2, D3 Street, Ward 25, Binh Thanh District \\ Ho Chi Minh City, Vietnam
}

\author{
Thi-Ngoc-Thanh Nguyen \\ Ho Chi Minh City University of Transport \\ No. 2, D3 Street, Ward 25, Binh Thanh District \\ Ho Chi Minh City, Vietnam
}

\begin{abstract}
Traffic congestion has become an important problem in recent years. The main reason is the increase in the population in big cities and respective increase in number of vehicles. Traffic jams not only affect the human routine lives but also lead to a rise in the cost of transportation. So, an automatic traffic control system is required to manage the traffic congestion problem. The traffic density analysis will support the traffic management problems such as intelligent traffic signal control, traffic planning, etc. This paper has proposed a traffic density analysis method based on image segmentation with adaptive threshold. The system was designed and evaluated with the traffic images taken in Ho Chi Minh City, Viet Nam. The proposed method provides a accuracy analysis rate higher than $97 \%$ and a verification error lower than $3 \%$.
\end{abstract}

\section{General Terms}

Traffic Density, Otsu threshold, Image Segmentation, Histogram

\section{Keywords}

Traffic Density, Otsu threshold, Image Segmentation, Histogram

\section{INTRODUCTION}

The calculation of traffic density is utilized for traffic control with different purposes. The density calculation helped in automatic traffic lights switching for better traffic management. A lot of researches and works have been done on traffic analysis using image processing techniques. The authors of [1] discussed a model to count the traffic load by some parameters such as edge detection, histogram equalization, labeling and removing the noise with the help of median filter. The authors of [2] has proposed an approach to road monitoring and traffic problem, such as vehicle tracking, speed measurement, jam detection and number-plate recognition. The authors of [3] has proposed automated vehicle detection based on average filter to reduce the noise effect. Thresholding value is applied to remove the unwanted objects other than the vehicle. The authors of [4] suggested algorithm to determine the number of vehicles on the road and to control the traffic by calculating density only on the target area. There are several other methods of identifying traffic density [5, 6], although these techniques are effective but the calculation is so complicated.

It found that a traffic image with very crowded traffic density, the traffic vehicles will appear most of image and a traffic image with sparse traffic density, the background will appear most of image. This paper based on image segmentation method to classify a traffic image into two classes including background and traffic vehicles with adaptive threshold. Each traffic image has a private adaptive threshold. The calculation of adaptive threshold was improved from Otsu threshold and histogram of grayscale traffic image. This paper was improved from our previous works [7] 8].
The rest of the paper is organized as follows. The theoretical background is discussed in Section 2. Section 3 presents the design of system architecture. In section 4 , the numerical results of experiment are illustrated. Finally, Section 5 concludes this paper and figures out the future works.

\section{THE THEORETICAL BACKGROUND}

\subsection{Color Image}

Color image [9, 10] is presented by a triple RGB (Red, Green, Blue). The value of color channels ranges from 0 to 255 . Set of 3 color channels will generate $2^{24}$ colors $(256 * 256 * 256$ colors $)$.

\subsection{Grayscale Image}

Grayscale images [9 10, 13] are the color images using RGB color system in which the Red, Green, Blue have the same light intensity. So, the grayscale image just need to use one light intensity to show each pixel. The gray level of grayscale image ranges from 0 to 255. There are some techniques to convert color image into grayscale image such as lightness with the equation $(\max (\mathrm{R}$, $\mathrm{G}, \mathrm{B})+\min (\mathrm{R}, \mathrm{G}, \mathrm{B})) / 2$, luminosity with the equation $0.21 \mathrm{R}+$ $0.72 \mathrm{G}+0.07 \mathrm{~B}$.

\subsection{Binary Image}

A binary image [9, 10, 14] is a digital image that has only two possible values for each pixel. Typically, the two colors used for a binary image are black and white. The color used for the object(s) in the image is the foreground color while the rest of the image is the background color.

\subsection{Histogram}

Histogram [11, 12, 13] is the chart that shows the frequency of occurrence of each gray level in an image. Calculating histogram of image is performed as follows:

(1) Building the pixel matrix of the grayscale image.

(2) From the pixel matrix of the grayscale image, building the frequency of pixel.

\section{SYSTEM ARCHITECTURE}

System architecture is depicted in Figure 1 and performed as follows:

(1) Get traffic image from disk or other resources.

(2) Converting traffic image into grayscale image.

(3) Converting grayscale image into binary image with adaptive threshold.

(4) Calculating vehicle area based on binary image.

(5) Calculating background area based on binary image.

(6) Calculating ratio between vehicle area and background area.

(7) Traffic Density Analysis based on ratio between vehicle area and background area. 


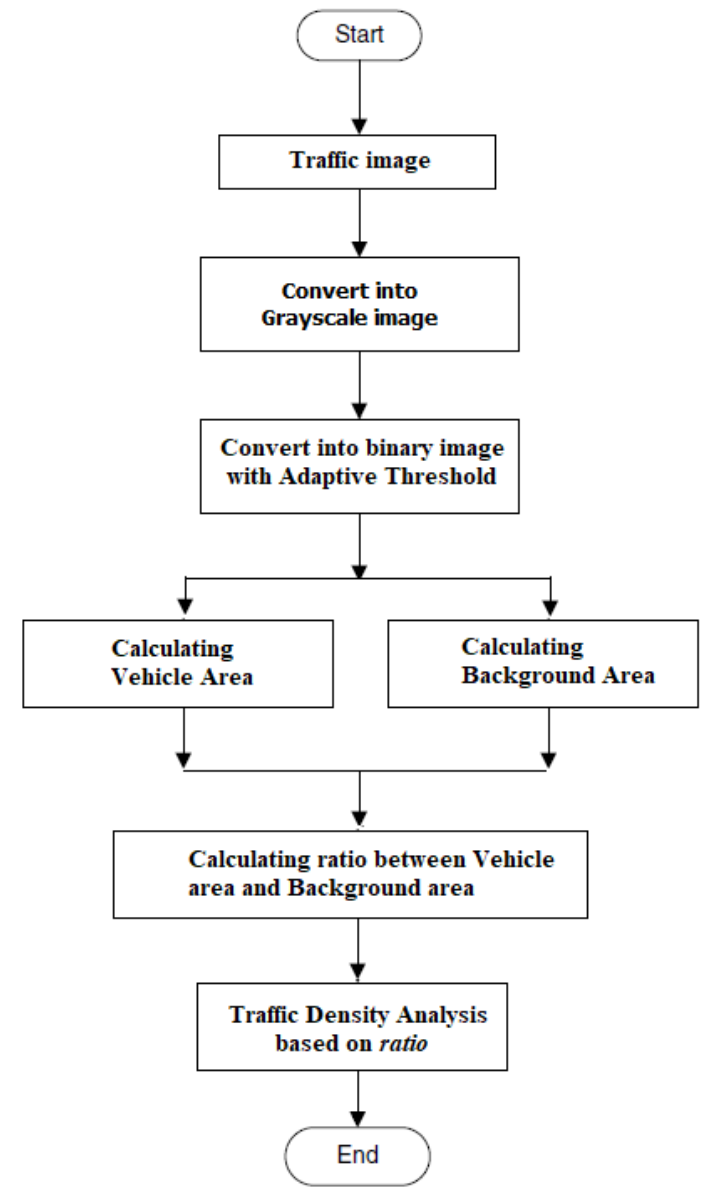

Fig. 1. The process of the training phase.

\subsection{Converting Traffic Image into Grayscale Image}

In this paper, the average method is selected, so the value of pixel in grayscale image is defined as (1).

$$
V=(R+G+B) / 3
$$

\subsection{Converting Grayscale Image into Binary Image with Adaptive Threshold}

The pixel value of grayscale image ranges from 0 to 255 . The equation (2) is used to convert grayscale image into binary image with threshold T.

$$
g(x, y)=\left\{\begin{array}{l}
0, f(x, y)<T \\
1, f(x, y)>=T
\end{array}\right.
$$

Using the fixed threshold is simple, but the result will be not effective. Figure 2 Figure 3 and 4 show color image, grayscale image and binary image with fixed threshold, respectively. Figure 5 and 6 show binary image with the value of threshold too high and too low.

It found that the traffic binary images will only include background and traffic vehicles. However, the traffic binary image has some details not fully displayed. Therefore, the problem is to find a method to get the optimal threshold aim to limit this problem.

In this paper, the adaptive threshold based on the histogram of grayscale image to determine. The adaptive threshold based on otsu threshold [15 16, 17] to determine as follows:

Gray levels of grayscale image range from 0 to L-1. Suppose that the image is separated into two categories $C_{0}$ and $C_{1}$ as

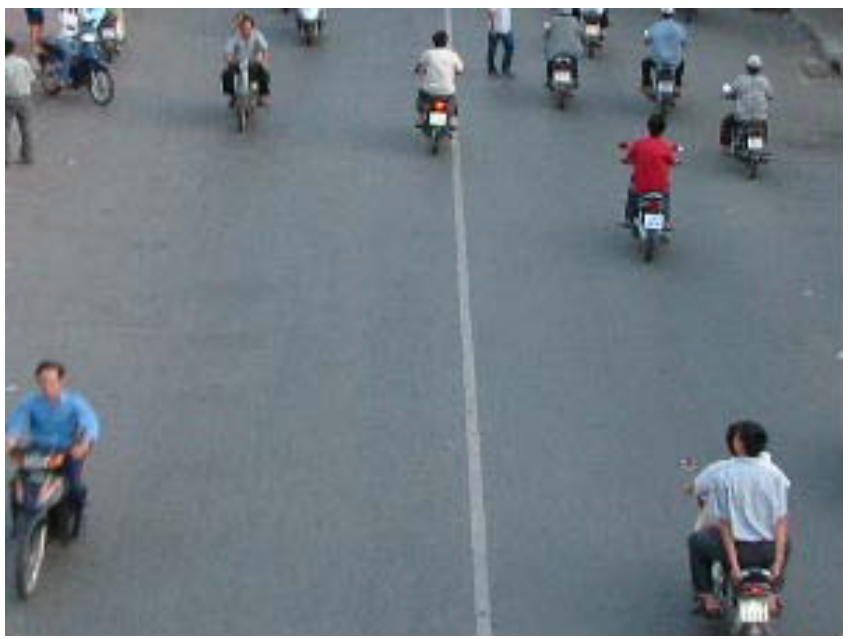

Fig. 2. Traffic color image

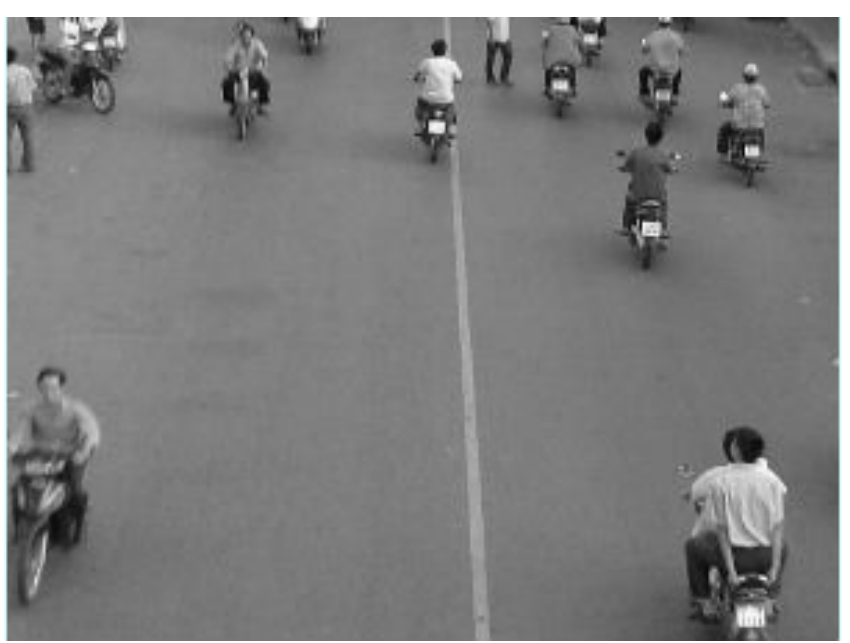

Fig. 3. Traffic grayscale image

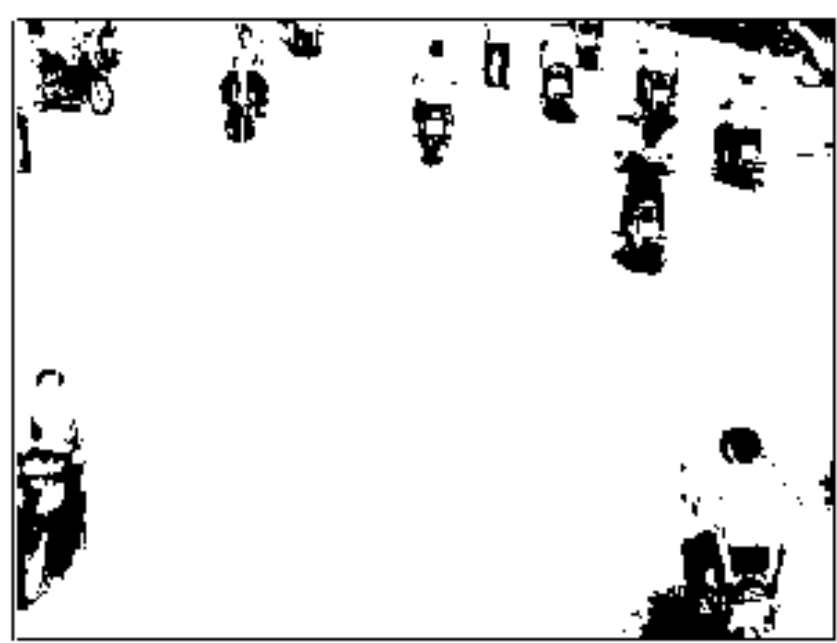

Fig. 4. Binary image with fixed threshold 

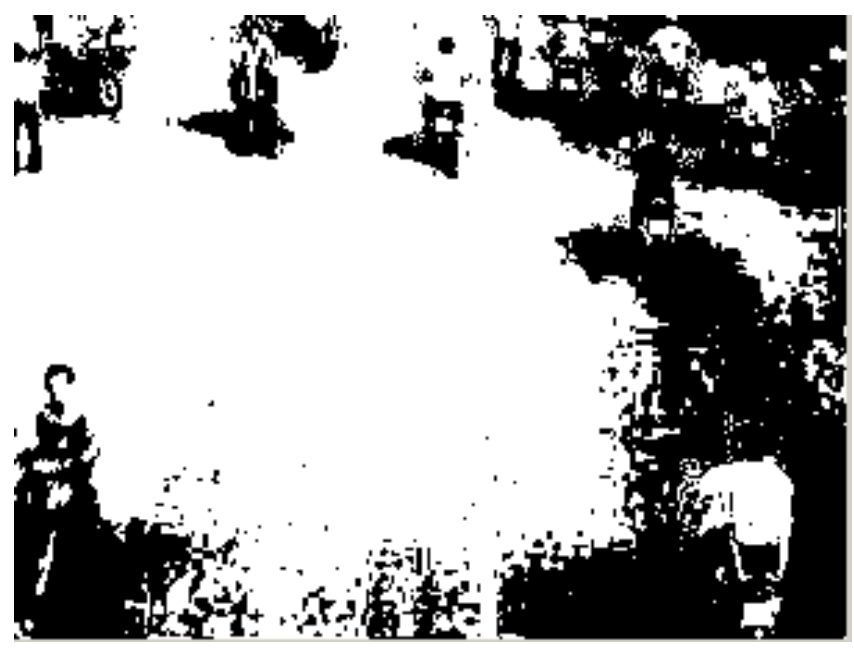

Fig. 5. Binary image with threshold too high

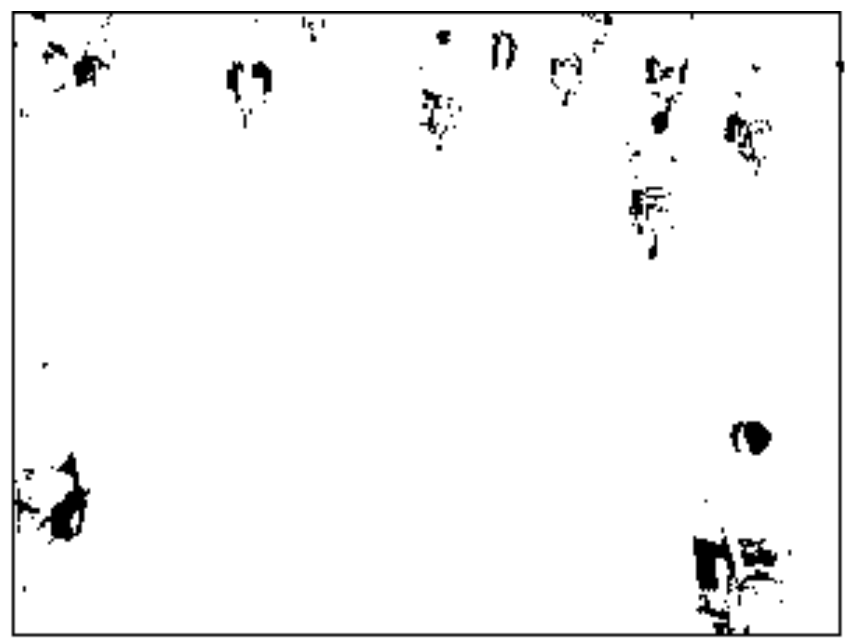

Fig. 6. Binary image with threshold too low

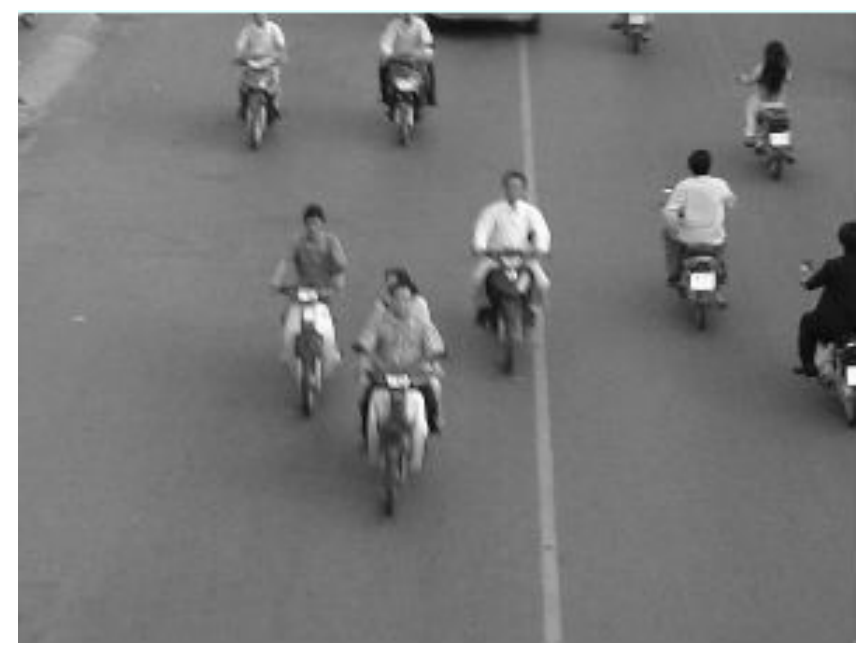

Fig. 7. Traffic grayscale image

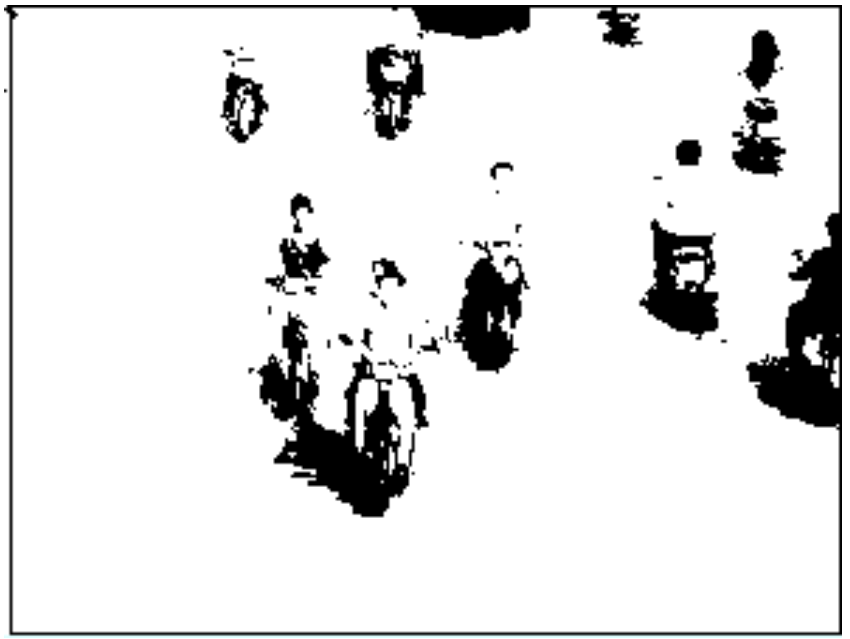

vehicles and background respectively consisting of threshold $\mathrm{T}$, then $C_{0}$ and $C_{1}$ correspondingly to the pixels whose gray levels are $\{0,1, \ldots, \mathrm{T}\}$ and $\{\mathrm{T}+1, \mathrm{~T}+2, \ldots, \mathrm{L}-1\}$. Some equations are calculated as follows:

- The probability at the $i^{t h}$ gray level is as defined 3 .

$$
P_{i}=\frac{n_{i}}{N}
$$

Where, $\mathrm{N}$ is the total of pixel in grayscale image, $\mathrm{N}=n_{0}+$ $n_{1}+\ldots+n_{L-1}$ and $n_{i}$ is the number of pixels at the $i^{\text {th }}$ gray level.

- The frequency value is as defined 4 .

$$
W=\sum_{i=0}^{T} P_{i}
$$

- The mean value is as defined (5).

$$
\mu=\frac{\sum_{i=0}^{T} i P_{i}}{W}
$$

- The total variance is as defined 6 .

$$
\sigma_{t}^{2}=\sum_{i=0}^{T}(i-\mu)^{2} P_{i}
$$

\section{Fig. 8. Binary image with threshold 94}

- The between-classes variance is the variation of the mean values for each class from the overall intensity mean of all pixels. It is as defined (7).

$$
\sigma_{b}^{2}=W_{0} W_{1}\left(\mu_{1}-\mu_{0}\right)^{2}
$$

Where, $W_{0}, W_{1}, \mu_{0}, \mu_{1}$ stand for the frequencies and mean values of two classes, respectively.

- The criterion function is as defined (8).

$$
\eta=\frac{\sigma_{t}^{2}}{\sigma_{b}^{2}}
$$

All possible threshold are evaluated in this way, and the one that maximizes $\eta$ is chosen as the optimal threshold.

For example, using the above mentioned algorithm to determine optimal threshold for grayscale image in Figure 7 Table 1 shows the value of criterion function with each gray level and 94 is optimal threshold. Figure 8 shows binary image with threshold 94.

\subsection{Calculating Vehicle Area and Background based on Binary Image}

Traffic binary image has only vehicles and background, the color used for vehicles is black and the color used for background is 
white. Vehicles area is calculated by counting the pixels with the non-zero value and background area is calculated by counting the pixels with the zero value.

\subsection{Traffic Density Analysis based on The Ratio between Vehicle Area and Background Area}

The ration between vehicle area and background area is is defined as 9 .

$$
\text { Ratio }=\frac{\text { Vehicles_Area }}{\text { Backgorund_Area }}
$$

\subsection{Traffic Density Analysis}

Traffic density based on the ratio between vehicle area and background area as follows:

- The ratio ranges from 0 to 0.25 : Sparse Traffic Density

- The ratio ranges from 0.25 to 0.5 : Normal Traffic Density

- The ratio ranges from 0.5 to 0.75 : Crowded Traffic Density

- The ratio ranges from 0.75 to 1 : Very Crowded Traffic Density

\section{EXPERIMENTAL RESULTS}

\subsection{Dataset}

In this paper, 300 traffic images were taken in Ho Chi Minh city, Viet Nam on many different streets with different times. These images with the traffic density such as very crowded traffic, crowded traffic, normal traffic and sparse traffic are experimented in proposed technique. 300 images are divided into 3 subsets, each subset contains 100 traffic images.

\subsection{Experiment Procedure and Results}

A system is designed by VB.NET programming language to experiment the proposed method with three subsets. The proposed method uses root mean square error (RMSE) [18] to measure accuracy. The correct analysis rates in percent are included in Table 2 Each result is the average of 100 runs, where we have randomly shuffled the traffic image in each run. Figure 9, 10 and 11 show the detail results of 100 runs. Figure 13, 14, 15 and 16 show some experimental results with four traffic densities.

\subsection{Comparing to other works}

The performance of Ref [7] and Ref [8] based systems in the same setup is lower than the proposed method. Table 3 shows

Table 1. The values of criterion

\begin{tabular}{|c|c|}
\hline \multirow{2}{*}{ Gray level } & $\eta=\frac{\sigma_{t}^{2}}{\sigma_{b}^{2}}$ \\
\hline 3 & 0.000300866239180 \\
\hline 4 & 0.000596484071193 \\
\hline$\ldots$ & $\ldots$ \\
\hline 28 & 0.183404567027206 \\
\hline 29 & 0.192019758479230 \\
\hline$\ldots$ & $\ldots$ \\
\hline 93 & 0.445166021994200 \\
\hline 94 & 0.445399906387494 \\
\hline 95 & 0.445369258716008 \\
\hline$\ldots$ & $\ldots$ \\
\hline 126 & 0.318034391145754 \\
\hline 127 & 0.315986641011137 \\
\hline$\ldots$ & $\ldots$ \\
\hline 254 & 0.000875942416999 \\
\hline 255 & 0 \\
\hline & \\
\hline
\end{tabular}

Table 2. The experimental results

\begin{tabular}{|c|c|c|}
\hline Subset & Analysis rates & Time for analyzing an image (ms) \\
\hline $1^{\text {st }}$ & $97.16 \%$ & 14 \\
\hline $2^{\text {nd }}$ & $97.07 \%$ & 11 \\
\hline $3^{\text {rd }}$ & $97.28 \%$ & 12 \\
\hline
\end{tabular}

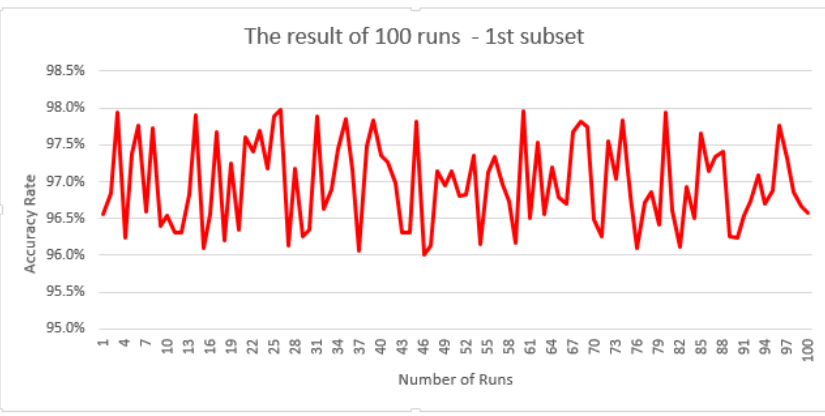

Fig. 9. The detail results of 100 runs - the first subset

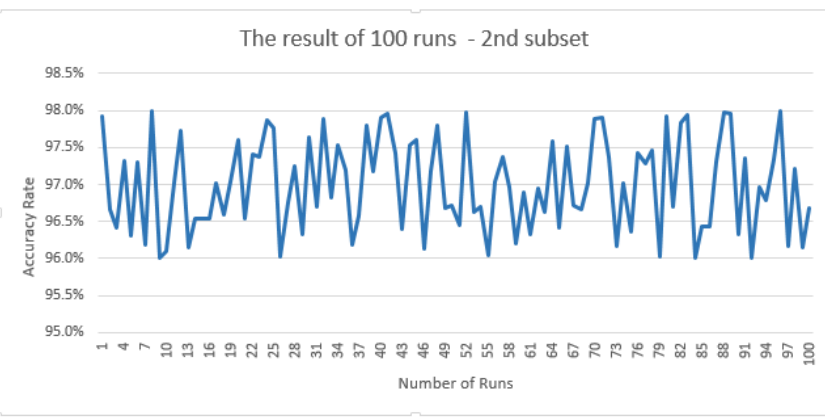

Fig. 10. The detail results of 100 runs - the second subset

the results of the comparison between the proposed method and the work Ref [7] and Ref [8] with the same three subsets. The results of the comparison are depicted in Figure 12 .

Table 3. The results of the comparison to other works.

\begin{tabular}{|c|c|c|c|}
\hline Subset & Ref [7] Technique & Ref [8] Technique & The proposed method \\
\hline $1^{\text {st }}$ & $91.02 \%$ & $96.23 \%$ & $97.16 \%$ \\
\hline $2^{\text {nd }}$ & $91.18 \%$ & $95.98 \%$ & $97.07 \%$ \\
\hline $3^{\text {rd }}$ & $91.09 \%$ & $96.14 \%$ & $97.28 \%$ \\
\hline
\end{tabular}

\section{ACKNOWLEDGMENTS}

The authors wish to thank reviewers for their reading of our manuscript and their insightful comments and suggestions.

\section{CONCLUSIONS}

In this paper, the traffic density analysis technique based on image segmentation with adaptive threshold has proposed. The technique is designed and experimented via Visual Studio with 300 images taken in Ho Chi Minh city, Viet Nam on many different streets with different times. The best result was obtained with $97 \%$ accuracy.

In the future, The proposed method will be improved by filtering to reduce the noise and increasing the contrast of image. We will experiment with many other datasets aim to increase the effectiveness of traffic density analysis further. 


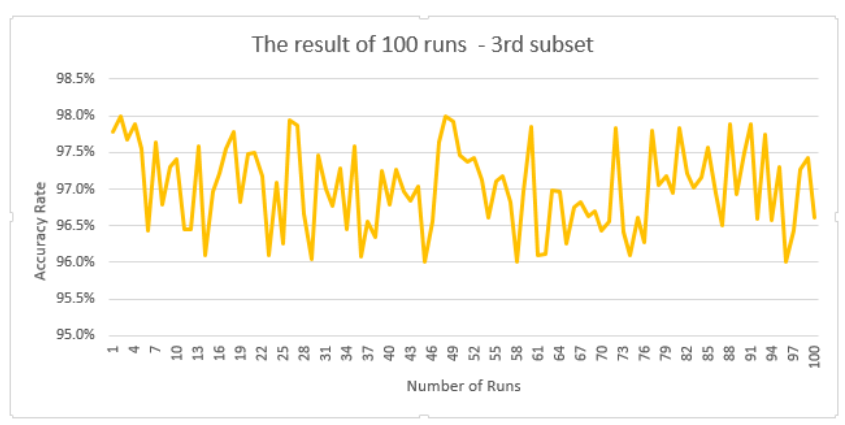

Fig. 11. The detail results of 100 runs - the third subset

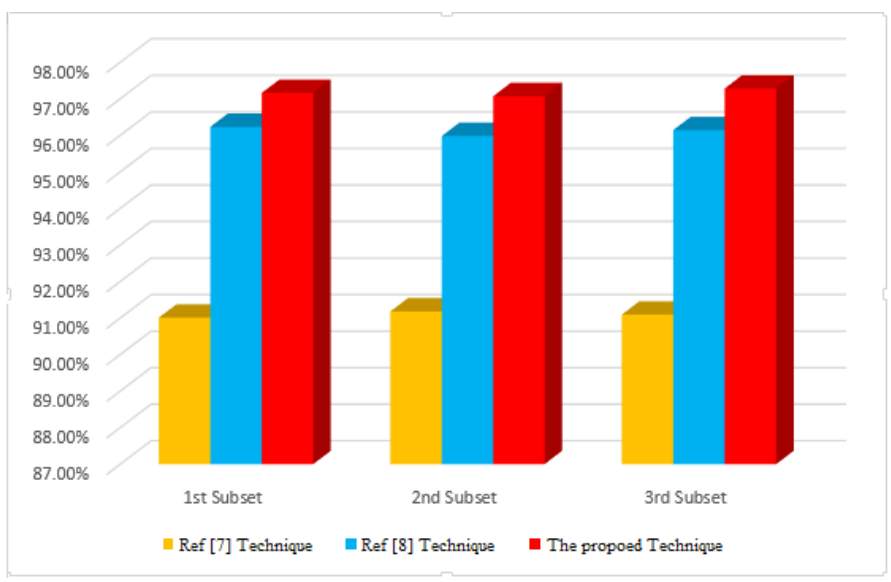

Fig. 12. The results of the comparison

\section{REFERENCES}

[1] Pratishtha Gupta , Purohit, Adhyana Gupta,Traffic Load Computation using Matlab Simulink Model Blockset International Journal of Advanced Research in Computer and Communication EngineeringVol. 2, Issue 6, June 2013

[2] Atkociunas, Blake, Juozapavicius Kazimianec Image processing in road traffic analysis Nonlinear Analysis: Modeling andControl, 2005, Vol. 10, No. 4, 315332

[3] Bharti Sharma, Vinoth Kumar Katiyar, Aravind Kumar Gupta, and Akansha Singh.(2014) The Automated Vehicle Detection of Highway Traffic images by Differential Morphological Profile. Journal of Transportation Technologies,4,150-156.

[4] Dharani.S.J, Anitha.V, Traffic Density Count by Optical Flow Algorithm using Image Processing,Automative Parts system and Application, ISSN 2347-6710(paper) Volume 3,Special Issue 2. April 2014

[5] Ozkurt C, Camci F. Automatic traffic density estimation and vehicle classification for traffic surveillance systems using Neural Networks. Mathematical and Computational Applications. 2009; 14(3):18796

[6] C. Stuiz, T. A. Runkler, Classification and Predicts of Road Traffic using Application Specific Fuzzy Clustering, Fuzzy Systems, IEEE Transactions, pp. 297-308, 2002.

[7] Luong Anh Tuan Nguyen and Thi-Ngoc-Thanh Nguyen. Traffic Image Classification using Horizontal Slice Algorithm. International Journal of Computer Applications 148(11):30-34, August 2016.

[8] Luong Anh Tuan Nguyen and Thi-Ngoc-Thanh Nguyen. Traffic Density Identification based on Neural Network and Histogram. International Journal of Computer Applications 172(9):8-13, August 2017.
[9] Al Bovik, Handbook of Image and Video Processing, Academic Press, 2000.

[10] Gonzalez, R., C., and Woods, R., E., 2001, Digital Image Processing, Prentice Hall, NJ, 2001

[11] Luong Anh Tuan Nguyen, Huu Khuong Nguyen. Traffic Density Identification Based On Histogram. Journal of Transportation Science and Technology, ISSN: 1859-4263, Vol 15-05/2015, pp 23-27.

[12] C. C. Sun. S. J. Ruan, M. C. Shie, T. W. Pai, Dynamic Contrast Enhancement based on Histogram Specification, IEEE Transactions on Consumer Electronics, 51(4), pp.13001305, 2005.

[13] Xiangyun Ye, Mohamed Cheriet, Senior Member, Ching Y. Suen (2001), Stroke-Model-Based Character Extraction from Gray-Level Document Images, IEEE, 2001.

[14] Binary Image (June, 2018), https : //en.wikipedia.org/wiki/Binary ${ }_{i}$ mage

[15] Otsu, N., "A Threshold Selection Method from Gray-Level Histograms," IEEE Transactions on Systems, Man, and Cybernetics, Vol. 9, No. 1, 1979, pp. 62-66.

[16] T. Bouwmans. Traditional and recent approaches in background modeling for foreground detection: An overview. Computer Science Review , 1112:31 66, 2014.

[17] Nida M. Zaitouna, Musbah J. Aqel. (2015). Survey on Image Segmentation Techniques. International Conference on Communication, Management and Information Technology (ICCMIT). pp 797806

[18] C. Willmott, and K. Matsuura, Advantages of the Mean Absolute Error (MAE) over the Root Mean Square Error (RMSE) in assessing average model performance, Clim. Res., 30, 7982, 2005. 


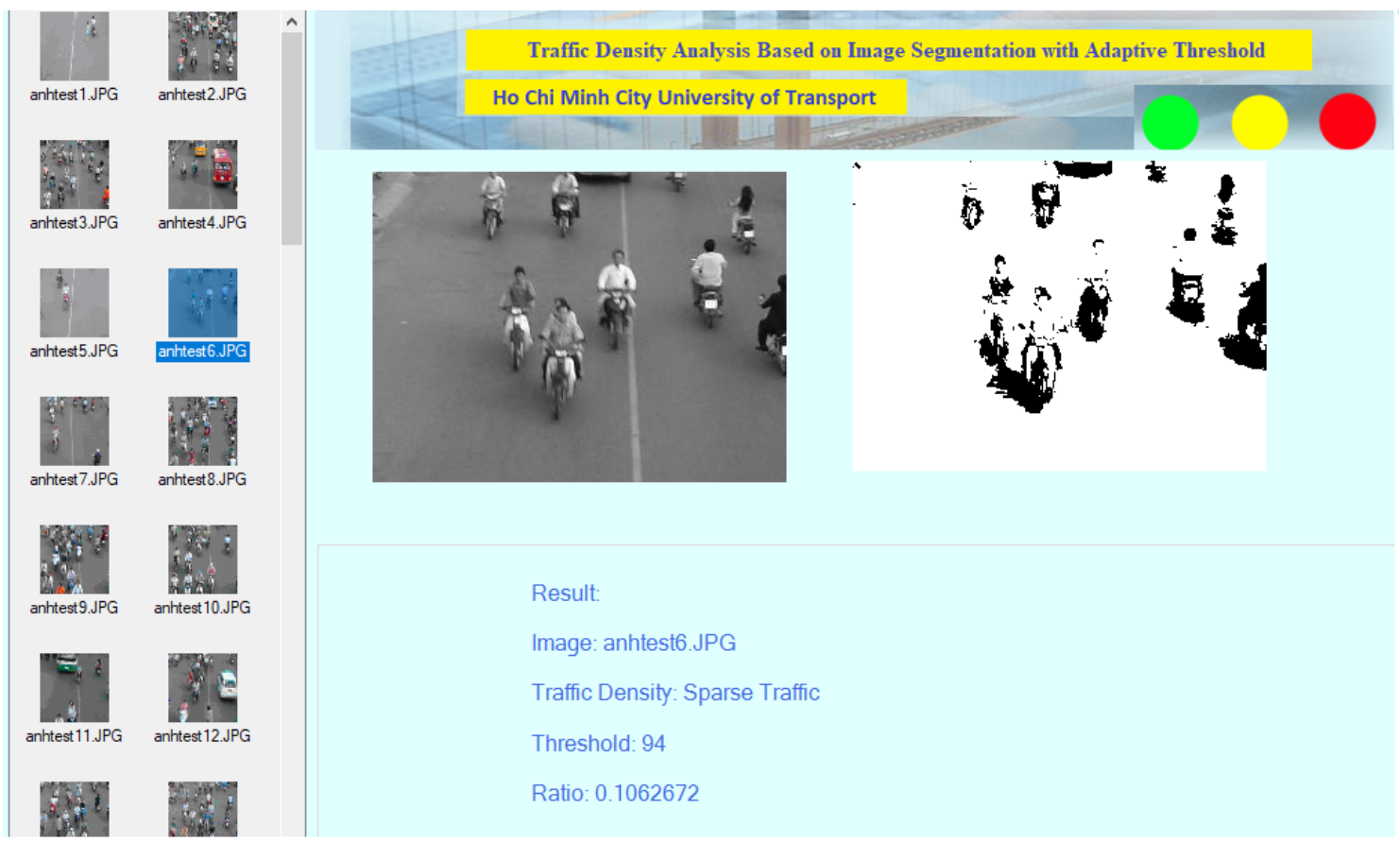

Fig. 13. The result of Sparse Traffic density

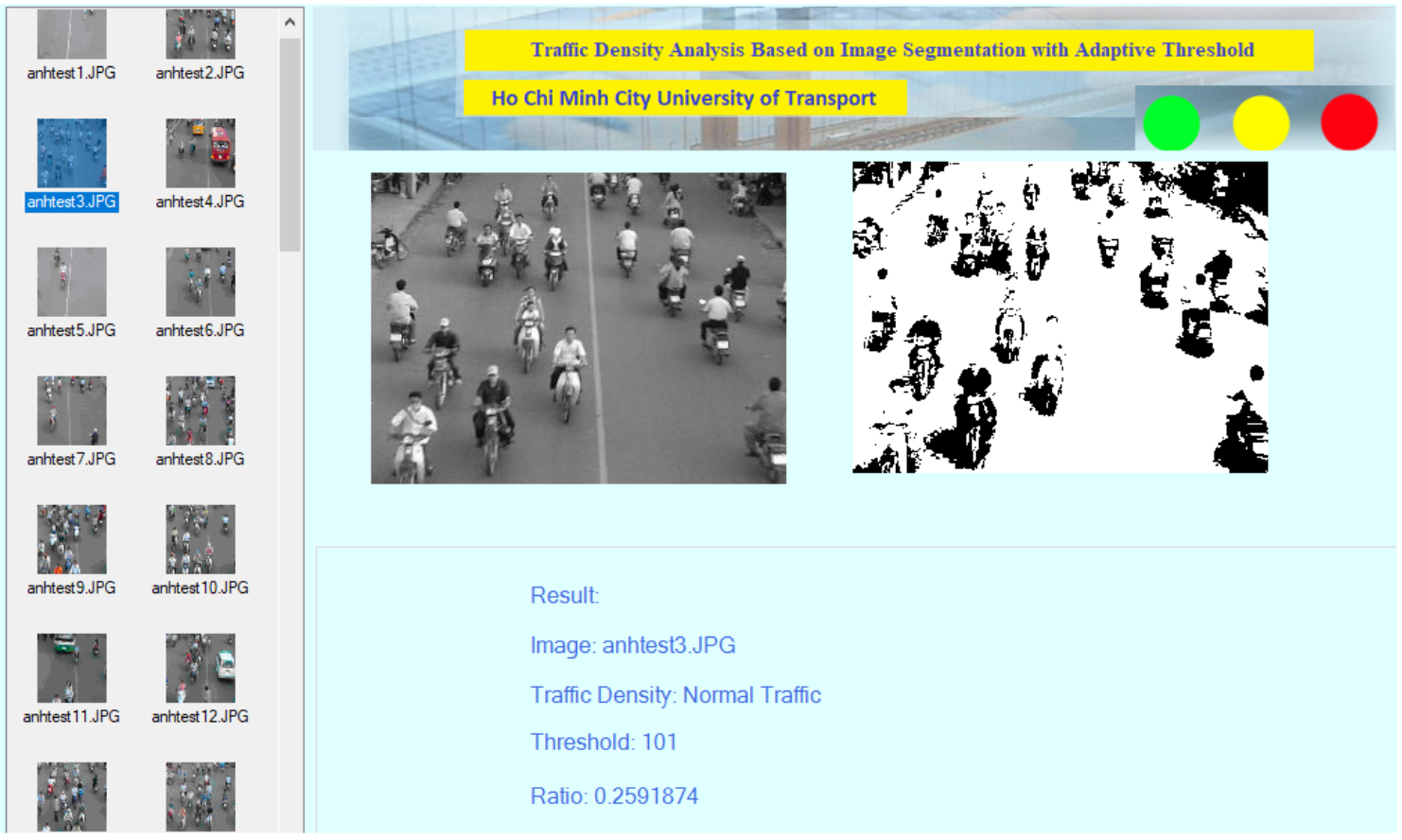

Fig. 14. The result of Normal Traffic density 


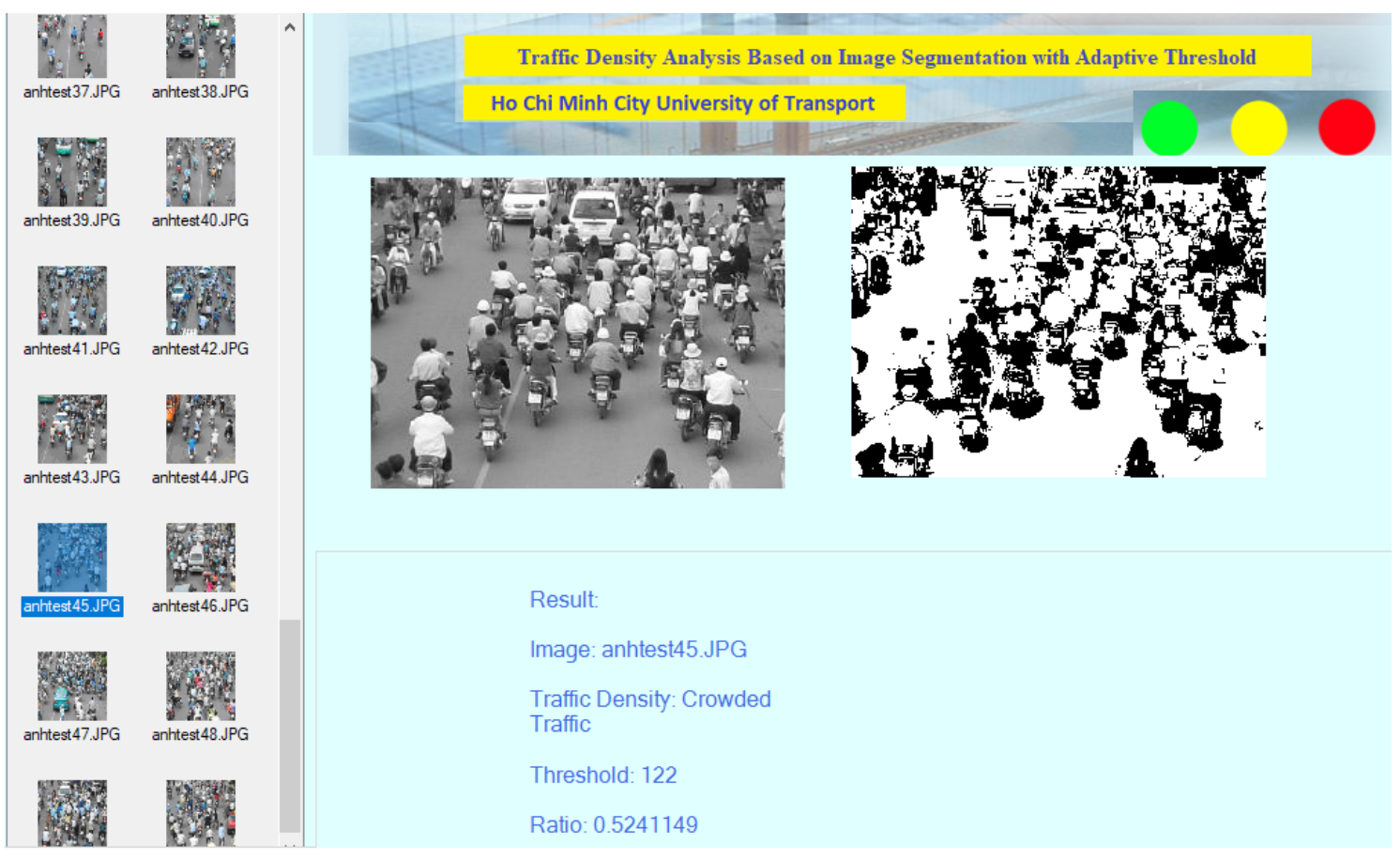

Fig. 15. The result of Crowded Traffic density

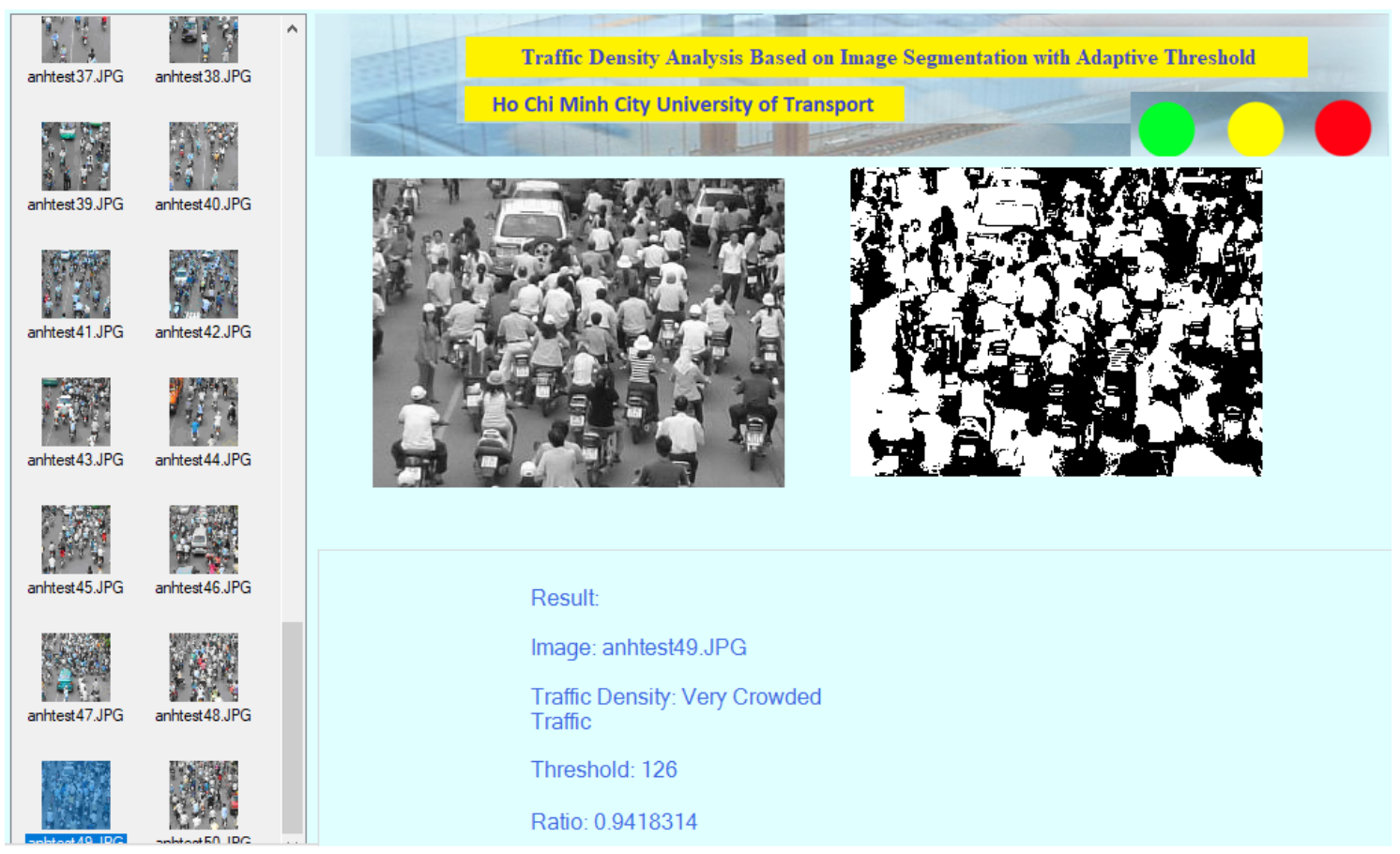

Fig. 16. The result of Very Crowded Traffic density 